\title{
No Evidence for Effects of a High-Frequency Repetitive Transcranial Magnetic Stimulation Series on Verbal and Figural Fluency and TAP Task Performance in Healthy Male Volunteers
}

\author{
Gerd Schaller Bernd Lenz Kerstin Friedrich Dominika Dygon \\ Tanja Richter-Schmidinger Wolfgang Sperling Johannes Kornhuber \\ Department of Psychiatry and Psychotherapy, Friedrich Alexander University of Erlangen-Nuremberg, \\ Erlangen, Germany
}

\section{Key Words}

Repetitive transcranial magnetic stimulation - Cognitive

function $\cdot$ Prefrontal cortex $\cdot$ Healthy volunteers

\begin{abstract}
Background: Study results on cognitive effects of repetitive transcranial magnetic stimulation (rTMS) in healthy people are inconsistent. Moreover, former trials performed exclusively single-session stimulations. This sham-controlled study analyzed the influence of 9 serial high-frequency rTMS on cognition. Methods: 44 young healthy male volunteers received active or sham rTMS. We evaluated verbal fluency tasks, the Ruff Figural Fluency Test and different Test for Attentional Performance tasks (alertness, go/no-go, divided attention, working memory, flexibility) prior to the first stimulation, immediately (within 5-30 min) after stimulation on day 5 and on day 10 (1 day after the last stimulation). Results: Overall, our statistical analyses revealed no significant cognitive effects of serial rTMS. Conclusion: In this sham-controlled study design, 9 serial rTMS over the left dorsolateral prefrontal cortex (targeted by the $5-\mathrm{cm}$ rule) did neither enhance nor impair the assessed cognitive functions in healthy male volunteers.

Copyright $\odot 2013$ S. Karger AG, Basel
\end{abstract}

\section{KARGER}

Fax +41613061234

E-Mail karger@karger.ch

www.karger.com
(C) 2013 S. Karger AG, Basel

0302-282X/13/0672-0069\$38.00/0

Accessible online at:

www.karger.com/nps

\section{Introduction}

Repetitive transcranial magnetic stimulation (rTMS) is a noninvasive tool for brain stimulation. High-frequency (HF) rTMS $(>1 \mathrm{~Hz})$ is known to induce effects by depolarization of neurons under the stimulating coil. The left dorsolateral prefrontal cortex (DLPFC) is a frequent target for HF rTMS. Selective cognitive improvements after HF rTMS over the left prefrontal cortex were described in past studies in patients [1,2] and healthy volunteers [3]. Nevertheless, considering the large number of investigations in this area, heterogeneous data were found. A recent review article evaluated the effects of HF rTMS over the left DLPFC on different cognitive domains in patients and healthy people. The authors state that rTMS most likely causes cognitive improvement when applied with $10-15$ successive sessions and $80-110 \%$ of motor threshold. Patients tend to reach greater improvement than healthy participants. However, many studies failed to demonstrate cognitive effects [4]. The few randomized sham-controlled studies analyzing the influence of single HF rTMS over the left DLPFC on cognition in healthy volunteers show inconsistent results. Huang et al. [5] ( $\mathrm{n}=$ 24) found no significant group differences in the Test for 
Attentional Performance (TAP) go/no-go task. Wagner et al. [6] ( $n=17$ men) observed a significantly retarded visual reaction time (RT) after active rTMS in the TAP divided attention task, whereas auditory RTs, the German version of the Stroop Test and the Computerized Wisconsin Card Sorting Test were not affected. Vanderhasselt et al. [3] ( $\mathrm{n}=28$ right-handed women) reported significantly decreased RTs in both (incongruent and congruent) trials of the Stroop Test following active rTMS and no influence on the Stroop interference effect and mood.

We recently reported a significant reduction of the Beck Depression Inventory (BDI) score of healthy young men during a 9-day series of active HF rTMS over the left DLPFC in a sham-controlled study design [7]. In this study, we investigate whether different cognitive functions associated with the DLPFC are improved by serial rTMS in healthy male participants in terms of neuroenhancement.

\section{Methods}

\section{Subjects}

We included 44 young healthy male volunteers after they had signed informed consent [7]. Exclusion criteria were severe organic or psychiatric disorders, long-term medication, abnormal laboratory tests and contraindications for rTMS [8]. Randomization to the active or sham group was performed using RANCODE 3.6 professional (IDV, Gauting, Germany). The study was carried out in accordance with the Declaration of Helsinki 1975 (revised in 1983) and the ICH-GCP Guidelines. Study protocol and informed consent were approved by the local Ethics Committee. We assessed handedness with the Shimizu Handedness Questionnaire [9].

\section{Neuropsychological Assessment}

We used the German version of verbal fluency (VF) tasks [10]. Participants were asked to say as many words as possible from a category (semantic or phonemic) in $2 \mathrm{~min}$. For semantic VF, animals (day 1), food (day 5) and professions (day 10) were employed as categories, whereas for phonemic VF, words beginning with the letter $\mathrm{p}$ (day 1), $\mathrm{m}$ (day 5) and $\mathrm{k}$ (day 10) were disposed as they are considered to be of equal difficulty. Total number of correct words and errors were statistically analyzed.

The Ruff Figural Fluency Test [11] is the nonverbal analog to VF tests. The challenge consists in drawing as many unique designs as possible within $60 \mathrm{~s}$ by connecting dots in different patterns. We used the total number of unique designs and the perseverative errors as outcome parameters.

The following tasks of the TAP computerized version 1.7 [12] were employed to quantify different neuropsychological parameters: alertness (4 series, 2 without cue stimulus, 2 with cue stimulus, ABBA design, 20 stimuli/series), go/no-go (condition 2: 2 critical stimuli out of 5,60 stimuli), divided attention [series 1 , condition c: simultaneous presentation of squares (100 stimuli) and tones (200 stimuli)], working memory (difficulty level 3: sec- ond to last number, 100 stimuli), and flexibility (condition 1: 'verbal', letters and numbers, mode 3: complex mode with alternating types of target stimuli, 100 stimuli). The software calculates different outcome parameters: RTs, correct reactions, omissions, anticipations, outliers, and errors. For detailed information on the applied tasks, refer to www.psytest.net and to Zimmermann and Fimm [13].

\section{rTMS Procedures and Study Design}

Stimulations were performed on 9 consecutive days with the following stimulation parameters: $100 \%$ motor threshold, $25 \mathrm{~Hz}$, 50 pulses/train, 15 trains/run, 8 -second intertrain intervals, and 750 pulses/session. We stimulated the left DLPFC, which was defined as the region $5 \mathrm{~cm}$ rostral to the point of optimal stimulation for the right abductor pollicis brevis muscle at a parasagittal plane in the left hemisphere [14]. Neuropsychological assessments were conducted prior to the first stimulation, immediately (within 5-30 min) after stimulation on day 5 and on day 10 (1 day after the last stimulation). It is well known that cognitive functions are subject to daytime variations. Hence, the individual's neuropsychological assessments were performed during the days' same time slots. The participants and the persons who directly administered the stimulation and rated were blinded to treatment allocation. The detailed stimulation procedures are described in our recent article on the effects of a 9-day series of rTMS on affectivity in healthy young men [7].

\section{Statistical Analysis}

In a first step, we analyzed the number of correct words of semantic and phonemic VF, the number of unique designs in the Ruff Figural Fluency Test and the median RTs of the TAP tasks. General linear models (GLMs; repeated-measures ANOVAs) were used to evaluate putative group differences between the active and sham group. Due to the number of analyzed outcome parameters in the first step $(n=10), p<0.005$ was considered statistically significant. Subsequently, we followed an exploratory approach and analyzed all other outcome parameters (table 1) using both GLMs and Student's t tests (or Mann-Whitney tests if the Kolmogorov-Smirnov test suggested no normal distribution). Subacute effects were defined as differences between day 5 and day 1 . Differences between day 10 and day 1 were interpreted as long-term effects. Data were analyzed using SPSS for Windows 19.0 (SPSS Inc., Chicago, Ill., USA).

\section{Results}

\section{Demographic Characteristics}

Detailed demographic data may be drawn from our recent article [7]. There was no significant difference in age (Student's t tests: d.f. $=42, \mathrm{t}=0.323, \mathrm{p}=0.748$ ) between the active (mean $=24.4$ years, $\mathrm{SD}=2.7$ ) and sham (mean $=24.1$ years, $\mathrm{SD}=2.9$ ) group. We performed evaluations regarding the whole population and right-handers $(<-12$ points in the Shimizu Handedness Questionnaire). Thirty-eight right-handers were included (21 in the active, 17 in the sham group). 
Table 1. Results of statistical analyses comparing all outcome parameters of the whole population (wp) between active and sham group

\begin{tabular}{llll}
\hline Test & $\begin{array}{l}\text { General linear models } \\
\text { between-subject effects (wp) }\end{array}$ & $\begin{array}{l}\text { Student's t tests/Mann-Whitney tests } \\
\text { subacute effects (wp) }\end{array}$ & $\begin{array}{l}\text { Student's t tests/Mann-Whitney tests } \\
\text { long-term effects (wp) }\end{array}$
\end{tabular}

TAP

Alertness without cue stimulus

Reaction time

Correct reactions

Omissions

Anticipations

Outliers

Alertness with cue stimulus

Reaction time

Correct reactions

Omissions

Anticipations

Outliers

Divided attention (square)

Reaction time

Correct reactions

Omissions

Anticipations

Outliers

Errors

Divided attention (tone)

Reaction time

Correct reactions

Omissions

Anticipations

Outliers

Errors

Go/no-go

Reaction time

Correct reactions

Omissions

Anticipations

Outliers

Errors

Working memory

Reaction time

Correct reactions

Omissions

Anticipations

Outliers

Errors

Flexibility

Reaction time

Correct reactions

Anticipations

Outliers

Errors

$$
\begin{aligned}
& \text { d.f. }=42, t=0.434, p=0.667 \\
& \text { d.f. }=42, t=1.330, p=0.191 \\
& U=231, Z=-1.000, p=0.317 \\
& U=221, Z=-0.989, p=0.323 \\
& \text { d.f. }=42, t=-1.330, p=0.191 \\
& \text { d.f. }=42, t=0.669, p=0.507 \\
& U=230.5, Z=-0.287, p=0.774 \\
& U=231, Z=-1.000, p=0.317 \\
& U=194, Z=-1.291, p=0.197 \\
& \text { d.f. }=36.459, t=-0.689, p=0.495 \\
& \text { d.f. }=42, t=0.737, p=0.465 \\
& \text { d.f. }=42, t=0.510, p=0.613 \\
& U=227.5, Z=-0.413, p=0.679 \\
& U=231, Z=-1.000, p=0.317 \\
& \text { d.f. }=42, t=-0.629, p=0.532 \\
& U=231, Z=-1.000, p=0.317 \\
& \text { d.f. }=42, t=0.945, p=0.350 \\
& \text { d.f. }=42, t=0.228, p=0.821 \\
& U=216.5, Z=-0.744, p=0.457 \\
& U=231, Z=-1.000, p=0.317 \\
& \text { d.f. }=42, t=-0.617, p=0.541 \\
& *
\end{aligned}
$$

$$
\begin{aligned}
& \text { d.f. }=42, \mathrm{t}=-0.423, \mathrm{p}=0.674 \\
& \text { d.f. }=42, \mathrm{t}=0.362, \mathrm{p}=0.719 \\
& \mathrm{U}=242, \mathrm{Z}=0.000, \mathrm{p}=1.000 \\
& \mathrm{U}=231.5, \mathrm{Z}=-0.564, \mathrm{p}=0.573 \\
& \text { d.f. }=42, \mathrm{t}=-0.362, \mathrm{p}=0.719 \\
& \\
& \text { d.f. }=42, \mathrm{t}=0.742, \mathrm{p}=0.462 \\
& \text { d.f. }=42, \mathrm{t}=-0.332, \mathrm{p}=0.742 \\
& \mathrm{U}=231, \mathrm{Z}=-1.000, \mathrm{p}=0.317 \\
& \mathrm{U}=202.5, \mathrm{Z}=-1.083, \mathrm{p}=0.279 \\
& \text { d.f. }=42, \mathrm{t}=0.165, \mathrm{p}=0.870
\end{aligned}
$$

d.f. $=42, t=0.515, p=0.609$

d.f. $=42, \mathrm{t}=1.374, \mathrm{p}=0.177$

$\mathrm{U}=227, \mathrm{Z}=-0.397, \mathrm{p}=0.691$

$\mathrm{U}=231, \mathrm{Z}=-1.000, \mathrm{p}=0.317$

d.f. $=42, \mathrm{t}=-1.760, \mathrm{p}=0.086$

$\mathrm{U}=242, \mathrm{Z}=0.000, \mathrm{p}=1.000$

d.f. $=42, t=0.468, p=0.642$

d.f. $=42, \mathrm{t}=1.606, \mathrm{p}=0.116$

$\mathrm{U}=218, \mathrm{Z}=-0.669, \mathrm{p}=0.503$

$\mathrm{U}=231, \mathrm{Z}=-1.000, \mathrm{p}=0.317$

$\mathrm{U}_{*}=216.5, \mathrm{Z}=-0.671, \mathrm{p}=0.502$

d.f. $=42, \mathrm{t}=0.470, \mathrm{p}=0.641$

d.f. $=42, \mathrm{t}=0.591, \mathrm{p}=0.558$

*

*

d.f. $=42, \mathrm{t}=-0.591, \mathrm{p}=0.558$

$\mathrm{U}=218, \mathrm{Z}=-0.647, \mathrm{p}=0.517$

d.f. $=42, \mathrm{t}=-0.742, \mathrm{p}=0.462$

d.f. $=35.361, t=-0.100, p=0.921$

$\mathrm{U}=198, \mathrm{Z}=-1.110, \mathrm{p}=0.267$

$\mathrm{U}=169, \mathrm{Z}=-1.951, \mathrm{p}=0.051$

d.f. $=42, \mathrm{t}=-0.616, \mathrm{p}=0.541$

d.f. $=42, \mathrm{t}=-0.229, \mathrm{p}=0.820$

d.f. $=42, \mathrm{t}=-0.165, \mathrm{p}=0.870$

d.f. $=42, \mathrm{t}=0.093, \mathrm{p}=0.927$

d.f. $=42, t=0.171, p=0.865$

\section{Verbal fluency}

Phonemic

$\mathrm{F}(1,41)=0.023, \mathrm{p}=0.881$

Number of correct words Errors

Semantic

Number of correct words Errors

$$
\begin{aligned}
& \mathrm{F}(1,41)=0.047, \mathrm{p}=0.829 \\
& \mathrm{~F}(1,41)=0.752, \mathrm{p}=0.391 \\
& \mathrm{~F}(1,41)=0.172, \mathrm{p}=0.681 \\
& \mathrm{~F}(1,41)=0.310, \mathrm{p}=0.581
\end{aligned}
$$

Ruff Figural Fluency Test

Number of unique designs

Perseverative errors
$\mathrm{F}(1,41)=3.558, \mathrm{p}=0.066$

$\mathrm{F}(1,41)=0.447, \mathrm{p}=0.507$ d.f. $=42, t=-0.523, p=0.603$
d.f. $=42, t=-1.238, p=0.223$
d.f. $=42, t=-0.220, p=0.827$
$U=210.5, Z=-0.812, p=0.417$

d.f. $=35.442, t=-0.630, p=0.533$

d.f. $=42, \mathrm{t}=-0.158, \mathrm{p}=0.875$ d.f. $=42, \mathrm{t}=0.842, \mathrm{p}=0.404$

d.f. $=42, \mathrm{t}=-0.271, \mathrm{p}=0.787$

d.f. $=42, \mathrm{t}=0.335, \mathrm{p}=0.739$

$\mathrm{U}=216, \mathrm{Z}=-0.662, \mathrm{p}=0.508$

d.f. $=42, \mathrm{t}=1.114, \mathrm{p}=0.272$

d.f. $=42, \mathrm{t}=0.103, \mathrm{p}=0.918$

For GLMs (tests of between-subject effects), the F value and $\mathrm{p}$ value are indicated. Subacute effects were calculated by differences between day 5 and day 1. Differences between day 10 and day 1 represent long-term effects. For Student's t tests d.f., $t$ value and $p$ value and for Mann-Whitney tests U value, $\mathrm{Z}$ value and $\mathrm{p}$ value are indicated. The asterisks indicate all values $=0$. 


\section{Neuropsychological Assessment}

Neither GLMs nor Student's t tests (or Mann-Whitney tests) revealed significant $(\mathrm{p}<0.005)$ effects when comparing the active and sham groups' outcome parameters (table 1). Hence, we found no significant results for the between-subject factor 'group' in the GLMs of all outcome parameters, either in the whole population (table 1) or the subgroup of right-handers (data not shown). In addition, no subacute or long-term effects were detected when comparing the active and sham groups' outcome parameters of all the tasks using Student's $t$ tests (or Mann-Whitney tests) in either the whole population (table 1) or the right-handers (data not shown). Online supplementary table 1 (for all online supplementary material, see www.karger.com/doi/10.1159/000343502) shows the mean values and standard deviations of all the outcome parameters on day 1 , day 5 and day 10 of the whole population.

\section{Discussion}

Investigations on cognitive effects of rTMS in healthy people report inconsistent results. This may be due to differences in parameters of stimulation, methods to target the DLPFC, variances in populations (e.g. gender, age, psychosocial level) as well as in timing and selection of tasks for cognitive assessment.

To our knowledge, this trial is the first to investigate the influence of a HF rTMS series on cognitive functions in healthy volunteers. We aimed at a comprehensive neuropsychological assessment covering a wide range of cognitive functions such as verbal and figural fluency which integrate executive functions, memory/working memory and psychomotor speed as well as different TAP tasks analyzing basic cognitive abilities. The very homogeneous sample of young healthy men which was chosen to detect even small effects and the sham-controlled study design can be considered as strengths of the study. The included number of individuals was comparable to studies on healthy volunteers with significant results $[3,6]$. Contrary to our hypotheses, we found no significant effect of serial HF rTMS on performance in VF tests, the Ruff Figural Fluency Test as well as performance speed and accuracy in the TAP alertness, divided attention, go/no-go, working memory and flexibility tasks. It would be easy to draw the conclusion from these results that serial rTMS has no effect on cognition in healthy volunteers. This interpretation, however, would be a misreading of the data, because our study design includes several limitations. For the stimulation, we chose a high frequency of $25 \mathrm{~Hz}$ and applied a relatively low number of 750 pulses/session. Comparable studies analyzing the influence of single HF rTMS over the left DLPFC on cognition in healthy volunteers were performed with 1,560 [3] or 1,600 pulses/session [5,6]. Coil placement in our study followed the $5-\mathrm{cm}$ rule [14], a standard procedure to detect the DLPFC. Recent studies on TMS coil placement suggest the need for a positioning method incorporating individual anatomical information [15]. Anyway, we decided to use the above-mentioned standard procedure to detect the left DLPFC since this is the method used in the majority of clinical trials evaluating the effect of rTMS on healthy and depressed people [4] and because of reasons of practicability in this study and in daily clinical routine. Considering the targeting methods for the DLPFC in previous investigations on healthy populations, Vanderhasselt et al. [3] used a magnetic resonance-guided method in contrast to other studies $[5,6]$ using the $5-\mathrm{cm}$ rule. Finally, it is important to state that this investigation on young males does not allow drawing conclusions for women or elderly people.

At first glance, it appears contradictory that there is a significant decrease in the BDI score [7] and no effect on cognition in the actively treated group. There are two major explanatory approaches. The first relates to the specific characteristics of our study sample. We mostly included university students who are said to suffer more often from depressive symptoms [16]. This might account for the rTMS effect on the BDI score. In contrast, the participants' cognitive performance was already outstanding at the onset of the study, which may possibly explain the lack of cognitive improvement following active rTMS. This is in line with findings of de Jong et al. [17], who suggested that high-performing subjects do not benefit from pharmacological neuroenhancement, but even deteriorate. The second explanation is that rTMS induces neurobiological effects which are remote to the directly stimulated area (i.e. rTMS of the left DLPFC modulates dopamine release in the ipsilateral anterior cingulate cortex [18]). Such alterations in brain regions associated with mood might underlie the observed BDI improvements. Furthermore, the lack of influence on cognitive performance in our study is in line with a recent study showing that TMS increases cerebral blood flow in the directly stimulated area without changes in a finger tapping task [19]. The authors of this study speculate that immediately occurring compensation mechanisms might account for the discrepancy. 
Considering the high interest in neuroenhancement $[20,21]$, we suggest reassessing the reported findings with different stimulation parameters (e.g. increased number of pulses/session) and coil positioning incorporating individual anatomical information in a larger and different sample.

\section{Acknowledgements}

Funding for this study was provided by the Department of Psychiatry and Psychotherapy, University of Erlangen-Nuremberg, Germany. We thank all volunteers for their participation and the reviewers for constructive suggestions.

\section{Disclosure Statement}

None of the authors had a financial or personal conflict of interest.

\section{References}

1 Moser DJ, Jorge RE, Manes MD, Paradiso S, Benjamin BS, Robinson RG: Improved executive functioning following repetitive transcranial magnetic stimulation. Neurology 2002;58:1288-1290.

2 Martis B, Alam D, Dowd SM, Hill SK, Sharma RP, Rosen C, Pliskin N, Martin E, Carson V, Janicak PG: Neurocognitive effects of repetitive transcranial magnetic stimulation in severe major depression. Clin Neurophysiol 2003; 114:1125-1132.

3 Vanderhasselt MA, De Raedt R, Baeken C, Leyman L, D'Haenen H: The influence of rTMS over the left dorsolateral prefrontal cortex on Stroop task performance. Exp Brain Res 2006;169:279-282.

4 Guse B, Falkai P, Wobrock T: Cognitive effects of high-frequency repetitive transcranial magnetic stimulation: a systematic review. J Neural Transm 2010;117:105-122.

5 Huang CC, Su TP, Shan IK, Wei IH: Effect of $5 \mathrm{~Hz}$ repetitive transcranial magnetic stimulation on cognition during a Go/NoGo task. J Psychiatr Res 2004;38:513-520.

6 Wagner W, Rihs TA, Mosimann UP, Fisch HU, Schlaepfer TE: Repetitive transcranial magnetic stimulation of the dorsolateral prefrontal cortex affects divided attention immediately after cessation of stimulation. J Psychiatr Res 2006;40:315-321.

7 Schaller G, Lenz B, Friedrich K, Dygon D, Richter-Schmidinger T, Jacobi A, Mueller SE, Maihöfner C, Sperling W, Kornhuber J: Repetitive transcranial magnetic stimulation influences mood in healthy male volunteers. J Psychiatr Res 2011;45:1178-1183.
8 Wassermann EM: Risk and safety of repetitive transcranial magnetic stimulation: report and suggested guidelines from the International Workshop on the Safety of Repetitive Transcranial Magnetic Stimulation, June 5-7, 1996. Clin Neurophysiol 1998;108:1-16.

9 Shimizu A, Endo M: Handedness and familial sinistrality in a Japanese student population. Cortex 1983;19:265-272.

10 Aschenbrenner S, Tucha O, Lange W: Regensburger Wortflüssigkeits-Test. Göttingen, Hogrefe, 2000.

11 Ruff RM/Deutsche Fassung von Feldmann B, Melcher P: Ruff Figural Fluency Test. RFFT. Leiden, PITS, 2004

12 Zimmermann P, Fimm B: Test for Attentional Performance (TAP), Computerised Version 1.7. Freiburg, Psytest, 1997.

13 Zimmermann P, Fimm B: Testbatterie zur Aufmerksamkeitsprüfung (TAP). Version 1.7, Handbuch Teil 1. Herzogenrath, Psytest, 2002.

14 Rumi DO, Gattaz WF, Rigonatti SP, Rosa MA, Fregni F, Rosa MO, Mansur C, Myczkowski ML, Moreno RA, Marcolin MA: Transcranial magnetic stimulation accelerates the antidepressant effect of amitriptyline in severe depression: a double-blind placebo-controlled study. Biol Psychiatry 2005; 57:162-166.

15 Peleman K, Van Schuerbeek P, Luypaert R, Stadnik T, De Raedt R, De Mey J, Bossuyt A, Baeken C: Using 3D-MRI to localize the dorsolateral prefrontal cortex in TMS research. World J Biol Psychiatry 2010;11:425-430.
16 Meier S, Milz S, Krämer A: Projektbericht, Gesundheitssurvey für Studierende in NRW (Laufzeit 11.2005-12.2007). Gesundheitslabor der AG Bevölkerungsmedizin und biomedizinische Grundlagen, Fakultät für Gesundheitswissenschaften, Universität Bielefeld, 2007. http://www.gesundheitsfoerdernde-hochschulen.de/Inhalte/F_Gesundheitssurvey_NRW/Projektbericht_GesSur_ NRW.pdf (accessed January 25, 2012).

17 de Jongh R, Bolt I, Schermer M, Olivier B: Botox for the brain: enhancement of cognition, mood and pro-social behavior and blunting of unwanted memories. Neurosci Biobehav Rev 2008;32:760-776.

18 Cho SS, Strafella AP: rTMS of the left dorsolateral prefrontal cortex modulates dopamine release in the ipsilateral anterior cingulate cortex and orbitofrontal cortex. PLoS One 2009;4:e6725.

19 Orosz A, Jann K, Wirth M, Wiest R, Dierks T, Federspiel A: Theta burst TMS increases cerebral blood flow in the primary motor cortex during motor performance as assessed by arterial spin labeling (ASL). Neuroimage 2012;61:599-605.

20 Larriviere D, Williams MA, Rizzo M, Bonnie RJ, AAN Ethics, Law and Humanities Committee: Responding to requests from adult patients for neuroenhancements: guidance of the Ethics, Law and Humanities Committee. Neurology 2009;73:1406-1412.

21 Franke AG, Lieb K: Pharmacological neuroenhancement and brain doping Chances and risks (in German). Bundesgesundheitsblatt Gesundheitsforschung Gesundheitsschutz 2010;53:853-859. 